\title{
Of Smarthomes, IoT Plants, and Implicit Interaction Design
}

\author{
Björn Bittner, Ilhan Aslan, Chi Tai Dang, and Elisabeth André \\ Human-Centered Multimedia Lab, Augsburg University \\ Augsburg, Germany \\ \{bittner, aslan, dang, andre\}@hcm-lab.de
}

\begin{abstract}
There seems to be a danger to carelessly replace routine tasks in homes through automation with IoT-technology. But since routines such as watering houseplants also have positive influences on inhabitants' wellbeing, they should be transformed through carefully performed designs. To this end, an attempt to use technology for augmenting a set of houseplants' nonverbal communication capabilities is presented. First, we describe in detail how implicit interactions have been designed to support inhabitants in watering their plants through meaningful interactions. Then, we report on a field study with 24 participants, comparing two alternative design implementations based on contrasting embodied interaction technologies (i.e., augmented reality and embedded computing technology). The study results highlight shortcomings of today's smartphone mediated augmented reality compared to physical interface alternatives, considering measurements of perceived attractiveness and expected effects on determinants of wellbeing, and discusses potentials of combining both modalities for future solutions.
\end{abstract}

\section{Author Keywords}

Experience Prototyping; User Study; Positive Computing

\section{CCS Concepts}

-Human-centered computing $\rightarrow$ Interaction design; Interaction design;

\section{INTRODUCTION}

New embedded technologies have been transforming how we, as home inhabitants, envision our everyday routines and modes of interactions with networked things in our future homes and buildings. While the automation of many routine tasks in the home is already possible and more automation may be part of a desirable future, Calvo and Peters [8] have criticized in their book "Positive Computing" an exclusive focus of technology design for productivity. They motivated a sensitivity for human wellbeing and potential as additional design goals.

Copyright (C) 2019 Association of Computing Machinery. This is the author's version of the work. It is posted here for your personal use. Not for redistribution. The definitive Version of Record can be found at: http://dx.doi.org/10.1145/3294109.3295618
Indeed, future scenarios seem to imply that inhabitants of smart homes won't have to care about anything but interact with everything in a magical manner and be well. However, increase in automation can be considered as a source for one of the main contemporary design challenges, with previous research arguing, for example, that autonomous technologies often lead users to feel a loss of control [4]. To make autonomous systems less obnoxious Ju and Leifer [26] have proposed to design human-like forms of interaction (i.e., combine explicit with implicit interactions) for which Wendy Ju's implicit interaction framework [24] provides support. Mennicken et al. [33] have recommended to focus on collaboration between inhabitants and homes as a direction to deal with the problems of automation. Ultimately, not all routine tasks in homes are viewed as "a waste of time" but some routines may even be considered healthy rituals. For example, Elings and Marjolein [15] found various studies from different countries that showed how interactions with plants promote human wellbeing. Thus, a routine task, such as watering the houseplants, should not be automated blindly, but designed carefully.

In this paper, we report on a series of design inquiries and a user study in the field with 24 participants, addressing the following research questions.

- How can we use IoT technology to support inhabitants in properly watering their plants without replacing the benefits inhabitants may have from interactions with their plants?

- Would inhabitants prefer related future interactive technology solutions to be based on Augmented Reality (AR) or purely embedded/physical technology in their homes?

We choose these two state-of-the-art modalities due to their contrasting nature, including how they may influence the use of space in the home. We also choose them to ensure ecological validity of our contributions. To address the research questions, we first explored possible implicit interactions with IoT plants based on experience prototyping methods [7], the Implicit Interaction Framework [24], and the Proxemic Interaction Framework [32]. Then we implemented exemplary designs for both interaction technologies and compared them in the field based on participants' perceived user experience and the relevant wellbeing determinants [8] autonomy, competence, and motivation through observations and questionnaires.

The primary contribution of our research is in providing answers to the research questions, but we hope it also provides knowledge, sensibility, and guidance to address in general the 
challenges of designing "healthier" routines in smarthomes and interactions with smart things in homes.

\section{BACKGROUND}

Automated watering of plants has been the subject of research for the last 30 years (e.g., $[42,11])$ and commercial products have also emerged [38]. Previous research on enhancing plants in smarthomes seems mainly concerned with automated irrigation based on wireless moisture or soil humidity sensors that determine the condition of plants [2, 27]. The goals are the efficient use of water; i.e., to avoid over-hydration and dehydration of plants [27] and to take into account environmental conditions, such as temperature and evaporation rate of water [27]. Other applications include the notification of inhabitants about moisture levels [45] or the use of robot flowers as an intermediate device for interaction with inhabitants [9].

\section{Design Approaches for Interaction with/in Smarthomes}

The aforementioned goals attribute to the functional and instrumental aspects of smarthomes [47] but neglect the sociotechnical aspects. It makes sense to irrigate plants automatically when inhabitants are not at home for a long time and certainly contributes to the peace of mind [6]. However, it should not be the goal of domestic technology to replace the experiences from daily habits and routines that inhabitants enjoy but enhance those home experiences with technology [19, 39]. The daily routine of watering plants belongs to such experiences because it includes not only watering but also caring for the plants in the context of human social behavior.

Therefore, the body of related work concerned with design approaches for interactions with and in the smarthome has been increasing. For example, Venkatesh [44] presented a theoretical framework that included and interlinked the key constructs social space and technological space. Following this framework, interactions with household-technology have to consider both perspectives. In contrast, Heath and Bell [19] promoted a practical approach; i.e., to apply scenario-based prototyping, which has already been applied with success for industry environments also for home scenarios.

Eggen et al. [13] explored people's home experience during interactive sessions with families. Based on the outcome of these sessions, they realized and studied multiple prototypes, which enable experiences that "go beyond supporting tasks". They emphasize that the design for home experiences requires "endusers to realistically interact with and experience an application in the proper", that is actual physical, social, and cultural, "context of use". Later, Eggen et al. [14] proposed a holistic approach that addresses the design of home experiences from a user-driven perspective, where the home experience is about emotions and not solely the physical smarthome technologies surrounding inhabitants. Their approach considers a deep understanding of basic human needs in the smarthome context, such as autonomy, competence, or relatedness. Based on these needs, they applied "state-of-the-art psychological theories" on human processes to "enhance everyday life and improve the well-being of people".

\section{Designing Interactions with Smart Things}

Kranz et al. [28] stated that one of the challenges of embedded interactions is whether implicit or explicit interactions should be used. The advantage of implicit interactions is that users can concentrate on their actual goals and are not aware of computer systems. Implicit interactions are thus a reasonable way to support users without having them to learn new interactions with the system and allowing them to water their plants as they usually would. According to Buchenau and Suri [7], Experience Prototyping is suitable to explore user interactions with future systems. Moreover, they argue that role-plays, as an Experience Prototyping technique, can be used to investigate behavior of users and artifacts, with the advantage that the artifacts' roles can also be played by people and thus insights on how smart things should act and react can be gained without having to go through a time and effort consuming software development process.

Wendy Ju suggests to transfer observed implicit interactions in role-plays over to the system design with the help of an Implicit Interaction Framework [24], which aims to support designers in analyzing and getting inspirations from interactions between a user and a non-human agent (e.g., [25, 31]). Another relevant framework for designing spatial forms of implicit interactions is the Proxemic Interactions Framework [32], which was introduced by Marquardt and Greenberg to help design/develop interactions with/in ubiquitous computing ecologies and intelligent environments (e.g., [3, 40, 29]). They promote to analyze system behavior based on measures, such as position, orientation, and identity of things and users.

\section{Technologies and Techniques to Augment Things}

Cho et al. [10] used an LED emoticon to display the overall state of a plant and Angelini et al. [1] extended this concept by a display that informed the user about humidity, light conditions, and temperature. Most of these projects used the concept of physical computing [37] to digitize the plants. Microcontrollers (e.g., Arduino) and sensors (e.g., humidity and light sensors) are used to measure the state of the environment and actuators (e.g., water pump, LEDs) are used to manipulate it.

An alternative to physical actuators is providing information through the augmented reality. According to Milgram [35], $\mathrm{AR}$ is closer to the real environment in a continuum between the pure real environment and the purely virtual environment. The body of related work in AR is large and it is not surprising that AR has also been explored in the context of smarthomes, where it has often been used to enable remote identification and control of things (e.g., a lamp) without having to move to the thing $[43,17,30]$. Often, AR usage is proposed to help/guide users during activities, such as using appliances [36], assembling things [41], machine maintenance [21], or repair [20]. A problem with embedded technologies and specifically physical actuators is the interface bottleneck. For example, Janlert and Stolterman [23] argue that holograms or augmented reality interfaces are conditional, while physical actuators require absolute and real space. Thus, AR and physical actuators seem orthogonal to each other considering their materiality. With the HoloLens [34], Microsoft demonstrated new possibilities of AR. Apple introduced its ARKit [22] frame- 
work and Google introduced ARCore [16]; both technologies contribute to making AR accessible on many state-of-the-art smartphones, and thus an alternative technology to physical actuators in homes.

This background section provided the state-of-the-art in the literature relevant to our work so as to highlight its novel contribution. Our work considers socio-technical aspects in the context of interaction with domestic technology. However, instead of exploring inhabitants' home experience (as proposed by Eggen et al. [13]) for the design of domestic technologies, we considered implicit interaction [24] and proxemics [32] in role-plays [7] for interaction design with domestic technologies. Furthermore, we compared the interaction modalities $\mathrm{AR}$ and physical LEDs for domestic routines (i.e., watering plants) in smarthomes which has not been done before.

\section{IMPLICIT INTERACTIONS WITH NON-HUMAN AGENTS}

We started the design process with exploring potential houseplant watering scenarios and conducting role-plays as an experience prototyping methodology. That is, we explored how users would interact with houseplant and a watering can and vice versa, considering household plants and the can as nonhuman agents, who can communicate their need for water (or in case of the can, their purpose to water plants who are in need of water) with human-like capabilities. We then analyzed interaction patterns which occurred during the role-plays with the help of the Implicit Interaction and the Proxemic Interaction Framework. Afterwards, we mapped the information that needed to be communicated by non-human agents to users to a data format, which could be implemented by state-of-the-art technology, such as a display. Figure 1 depicts the general design process and the three main steps which we will describe in the following.

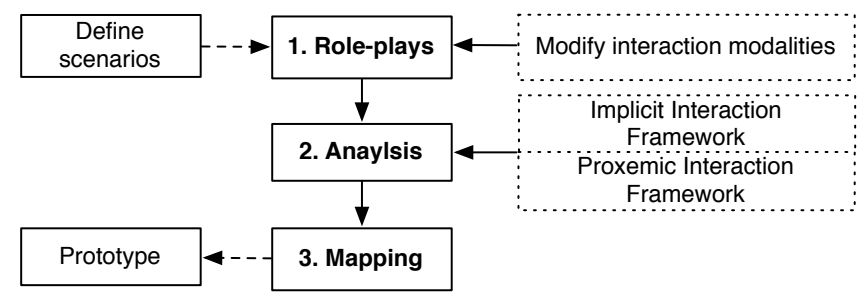

Figure 1. Overview of design process.

\section{Role-Plays}

We organized two rounds of role-plays across two days. Within each round, multiple scenarios were played in a real home.

\section{First Round of Role-Plays}

For the first round, we recruited four participants. One participant played the user, another one played the watering can and each of the remaining participants played a plant. While we had a set of predefined scenarios for the first round, we discussed together with the participants which of the scenarios were useful and played them out afterwards. In total, we played seven simple scenarios in the first round. For example, in one scenario only one plant required water and in another scenario two plants had to be watered by the user. The roleplays quickly revealed situations where the interactions were blurry and unclear. For example, a plant indicated by raising its arms that she needed water and the user came to her to water her. When the user stood next to her, she lowered her arms, confusing the user and not knowing if she still needed water. In order to investigate these unclear interactions more closely, a second round of role-plays was conducted.

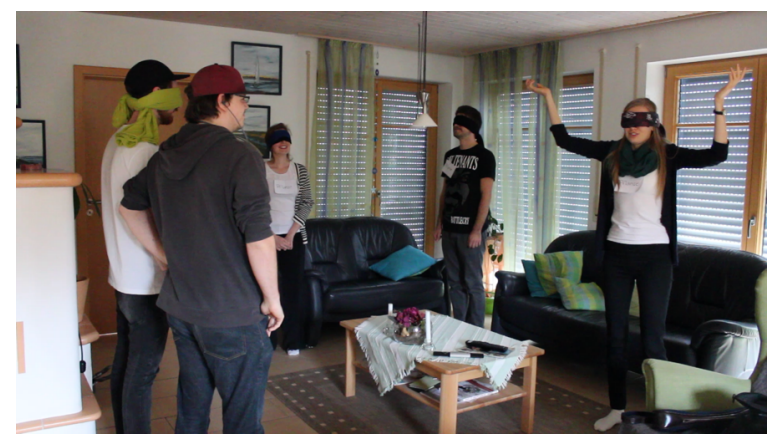

Figure 2. Scene from the second role-play.

\section{Second Round of Role-Plays}

The focus of the second round were four scenarios, each of which was intended to answer a specific question. A total of six participants were recruited to take part in the role-plays. While in the first round one researcher took part in some of the role-plays, in the second round researchers did not participate actively in role-plays. They just gave instructions at the beginning and then observed the participants during the execution. After each scenario, all participants were asked in a semistructured interview how they perceived everything, whether there were problems, or they noticed something interesting.

Similar to the first round of role-plays, one participant played the role of the user and one played the role of the watering can. The remaining four participants played plants, with three scenarios involving only three plants. The participants rotated in their roles so that information could be collected for each role from different perspectives. At the beginning of each scenario, each participant was given a personal goal in a oneon-one interview. The user was supposed to water the plants that needed water, the watering can was supposed to help the user and the plants were supposed to make sure that they received water when they needed it.

Scenario 1: all participants, except the role of the user, were blindfolded to foster communication, as shown in Figure 2. The plants and the watering can (i.e., the "non-human agents") were not able to see what was happening and had to talk to each other to exchange relevant information.

Scenario 2: the "non-human agents" used smartphones to chat with each other because a plant was in an extra room. The group had to direct the user's attention to this plant.

Scenario 3: the plants were given glasses and the watering can was given a real watering can. The plants had the task of receiving a certain amount of water in order to investigate the particular interaction more closely when the plants tell the user how much water they need.

Scenario 4: one of the plants played a defect plant demanding more and more water. 
The interviews conducted after each scenario were analyzed after the end of the role-play with a thematic analysis [5]. The idea was to see which interactions were understood by the other participants and in which cases the participants had problems to understand.

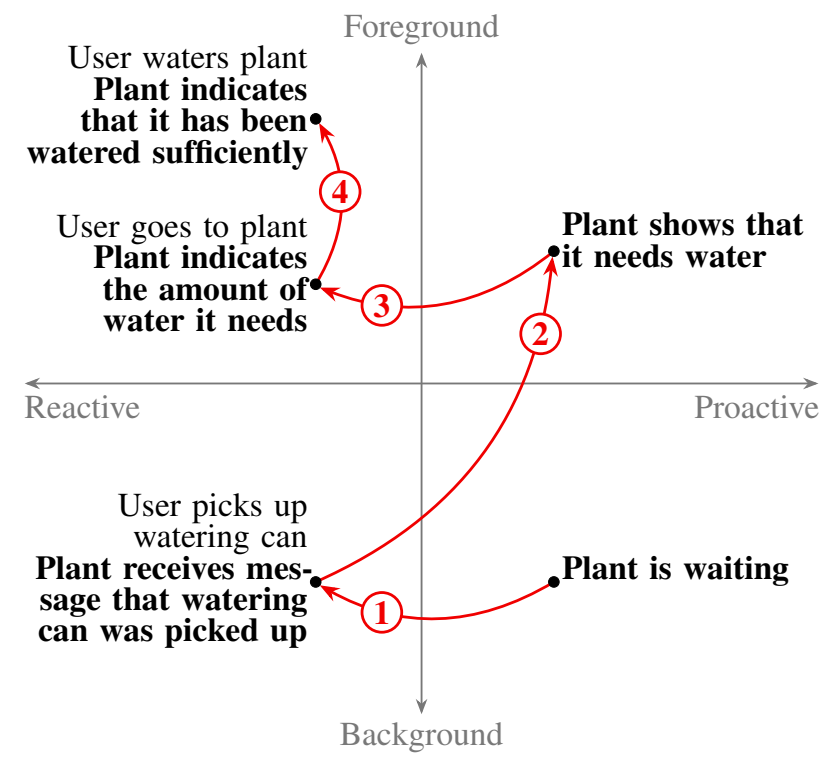

Figure 3. Interaction pattern between a user and a single plant, displayed with Implicit Interaction Framework.

\section{Analysis of Role-Plays}

After the role-plays, the interactions between users and the "non-human agents" were analyzed using the Implicit Interaction Framework. The watering process could be divided into three steps. Initially, the plants indicated that they needed water as soon as the user picked up the watering can. In the role-plays, the plants raised their arms or crouched together to visually symbolize "drying out". In the second step, the plants indicated exactly how much water they needed after the user walked with the watering can to the plant. The participants indicated with their fingers how far their glass should be filled. As soon as the plants had received enough water, they symbolized their satisfaction by nodding their heads or raising their thumbs. The interviews with the users revealed that users explicitly requested this confirmation at the end to ensure that they had watered enough. Figure 3 shows the interaction in the Implicit Interaction Framework with a single plant as defined after analysis of the role-plays.

Besides the interaction between user and plants, an interaction between user and watering can could also be observed. While the user used the watering can mostly to water the plants, a situation appeared in which the watering can tried to prevent the user from watering the plant by shaking.

After investigating role-play interactions, considering the implicit interaction Framework, the Proxemic Interactions Framework was used to explicitly investigate the spatial relationships between user and non-human agents. When the user took the watering can with their hand or entered the room with the watering can in their hand, the plants indicated whether they needed water. As soon as the user focused on a plant by approaching it and orientating towards it, the plant indicated exactly how much water it needed. When the user put the watering can down or left the room, the plants stopped displaying anything.

During the role-plays, we have also observed that users often did not know whether they had watered all the plants sufficiently. The plants tried to clarify among themselves whether all were satisfied, but they didn't inform the user. The interviews afterwards showed that the participants associated this task with the watering can. Consequently, we defined that the can should display an overall status of all plants in the room for the user as soon as the user picks up the watering can. We also defined that when the user changes the room, the status of the plants in the new room should be displayed until the user puts the watering can down.

\section{Mapping of Results}

The last step in the design process was to map the observed behavior patterns of people in the role-plays to information, which can be implemented in different technologies and modalities. For example, (i) plants must be able to visually indicate whether they need water and (ii) plants need to indicate how much water they need. Figure 4 depicts how we mapped the required information for both modalities (i.e., physical actuators and AR). We chose three different colors, each representing a status of the water level: red - very low; blue - low; green sufficient. The design is also capable to indicate whether the water level is between a minimum and a maximum mark. The optimum lies in the middle between the two markings.

The watering can needed, for example, to communicate (iii) that a plant did not require (more) water, and (iv) to display the status of the group of plants in the same room. We decided to use a vibration function for (iii) and either an LED or the smartphone's display for (iv). The display/LED on the can was used to display the worst state of all plants in the room (i.e. $\mathrm{red} / \mathrm{blue}$ as long as at least one plant needs water and green as soon as all plants have enough water). The watering can would be able to vibrate in case a plant did not need any water or had already received enough water (which would also be visualized directly by the plant's LEDs turning red).

\section{IMPLEMENTATION OF FUNCTIONAL PROTOTYPES}

Figure 4 depicts the plants and the watering can and how we augmented them either with LEDs or AR to visually communicate with users. Both types of augmentations were designed to provide the functionality for a user study to be conducted as a "Wizard-of-Oz" experiment. Therefore, for example, the water levels of the plants were manually configured and not measured using sensors. Each system included multiple plants and a watering can. The communication protocol MQTT (Message Queue Telemetry Transport), which is a typical IoT protocol, was used to implement the communication between the components of the systems, for which a server (called MQTT Broker) was also required. In addition, a smartphone was used to display the virtual objects of the AR prototype. Figure 5 presents the connections/relations between each thing and the user. 

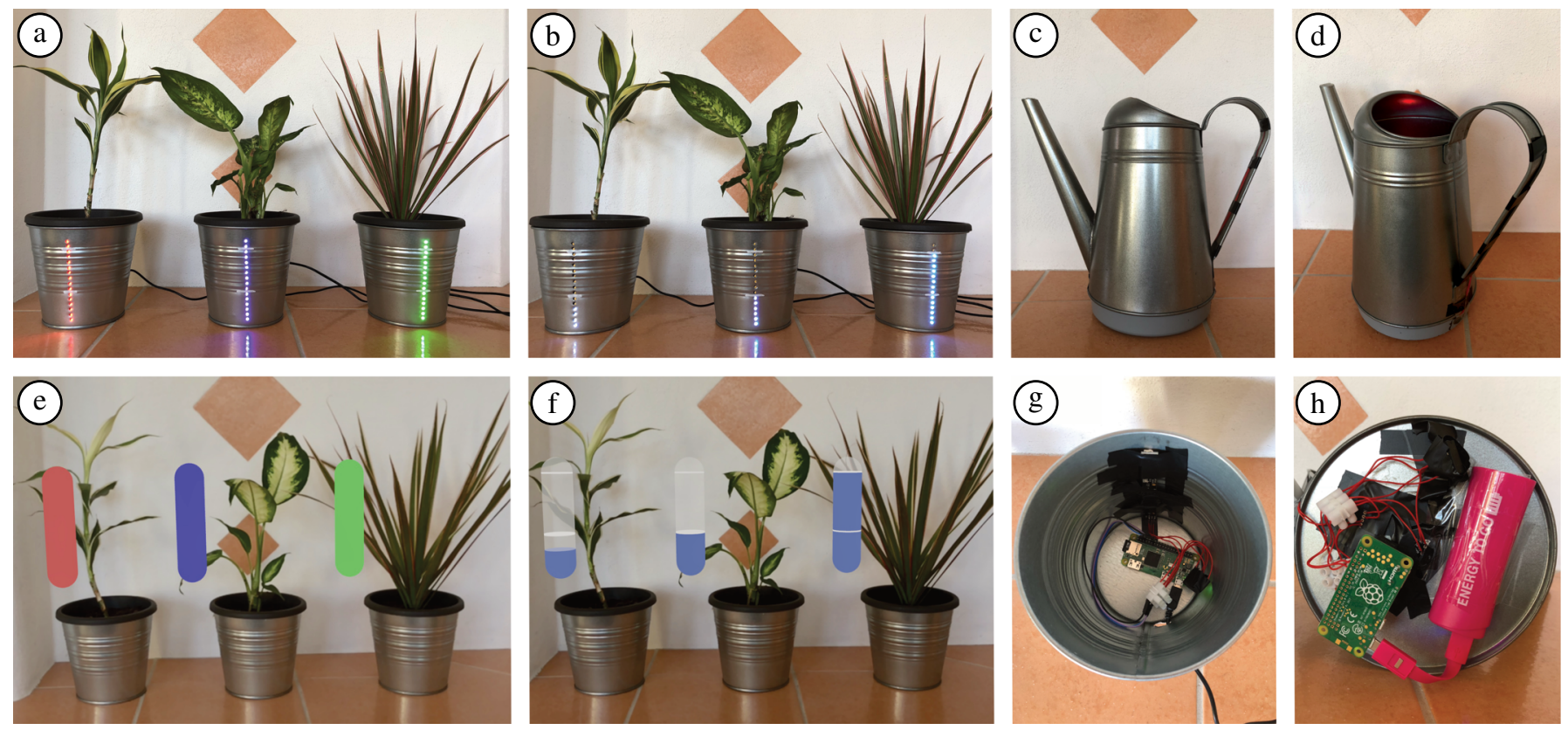

Figure 4. Overview of non-human agents and how they were augmented with embedded technology or Ar. a) and e) depict color codes, which were used to signal need for water. b) and f) depict how plants provided continuous feedback considering the amount of water they had received. c) and d) depict the watering can which was augmented with an LED and a vibration motor on the handle. g) and $h$ ) depict the inside of the a plant pot and the watering can, showing the Raspberry Pi Zero Ws.

\section{Watering Can}

A regular watering can was extended below the bottom with a Raspberry Pi Zero W and LEDs. Holes were drilled in the bottom of the watering can and closed with transparent material so that the LEDs could shine into the watering can from below (see Figure $4 \mathrm{~d}$ and $4 \mathrm{~h}$ ). The LEDs are used in the LED system to display the overall status of all plants in the room, while they are not used in the AR system because the status is displayed in the AR application. A vibration motor was attached to the handle, which can give users haptic feedback while holding the watering can, e.g. to give a warning when the user pours too much water. An acceleration sensor attached to the can detects when the user tilts the watering can to water the plants and sends a message to the plants every second as long as it is tilted. The hardware is powered by a battery.

\section{Plants}

Figure 4 shows plants of each of the two type of prototypes. The plants of the LED prototype also use a Raspberry Pi Zero W, which is located underneath the plant in the pot (see Figure $4 \mathrm{~g}$ ). The pot has 16 LEDs arranged one above another, which are used to communicate information to the user. The plants of the AR prototype do not use any displays, as information is visible via the smartphone screen. The water level of the plants is reported with a value ranged between 0 and 16 and is communicated to the user with the LEDs (see Figure $4 \mathrm{~b}$ ) or AR (see Figure 4f). Ideally, the water level should be between the two white markings (6 and 14). If the water level is lower, the plant urgently needs water, if it is higher, it has too much water and the user is warned if he tries to water the plant anyway. If the plants communicate their need for water after the user has raised the watering can and has not yet approached the plant, all LEDs (respectively the whole 3D

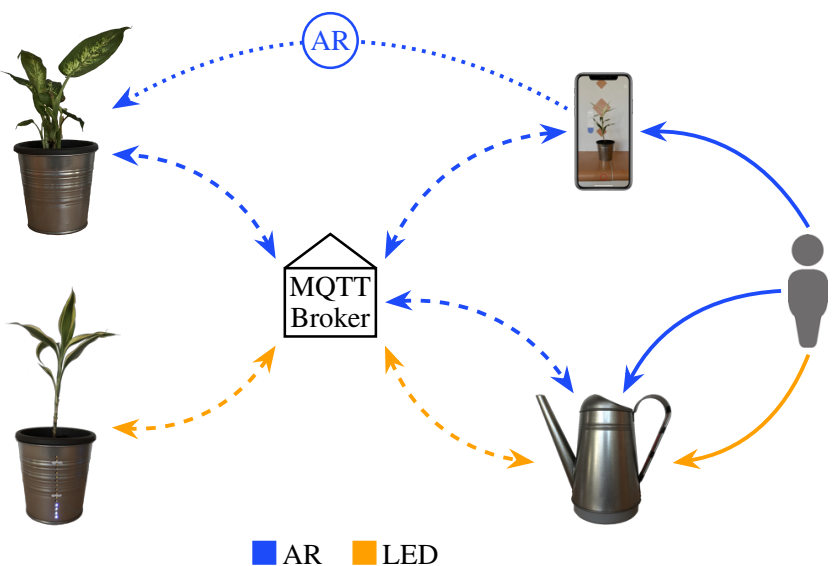

Figure 5. Setup of the system of the both modalities with a LED plant (bottom left) and a AR plant (top left). The arrows show which devices the user uses with the respective system (continuous arrows) and how they communicate with each other (dashed arrows) and how they are augmented via $\mathrm{AR}$ (dotted arrows).

model in the AR condition) light up in the same color (see Figure $4 \mathrm{a}$ and $4 \mathrm{e}$ ).

The plants of the LED prototype are not able to detect whether a user is approaching or not. Hence, in order to simulate proxemics, an application (as part of the "Wizard-of-Oz" research technique) has been developed that informs the plants when the user approaches. Moreover, the plants of both prototype types were also unable to determine whether they had just been watered. This is realized by messages from the watering can to the plants, which increase their water level by one level for each message. 

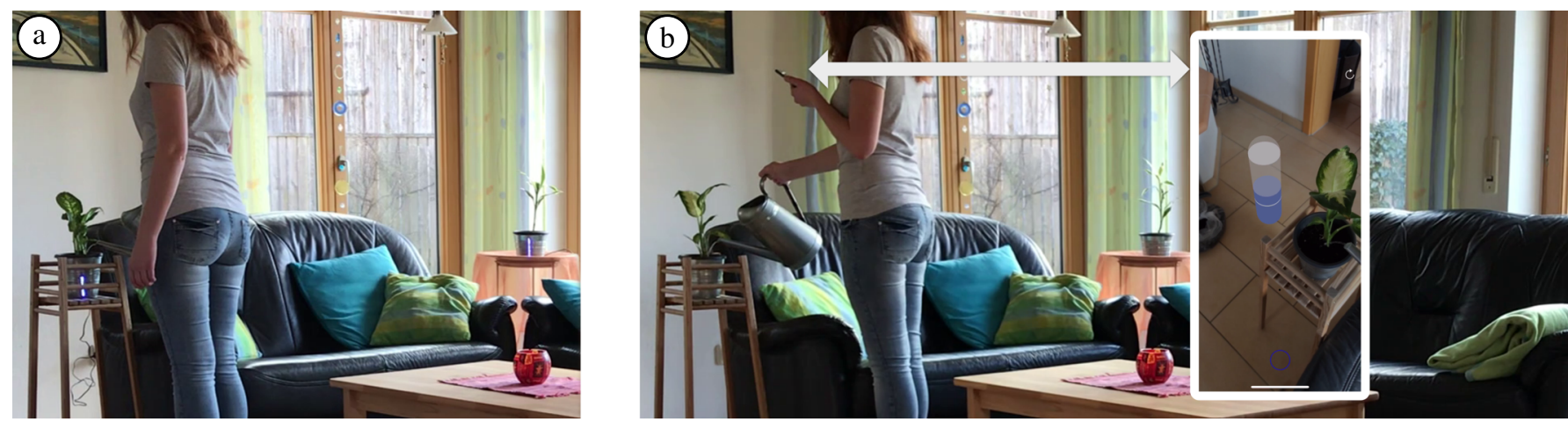

Figure 6. Screenshots of the user study. With a) showing a participant testing the LED system and b) showing a participant testing the AR system with the participants view of the AR through the smartphone display.

\section{AR Application}

To be able to display the information with AR, we developed a smartphone application using Swift and ARKit. After starting the application, the virtual information objects must be manually placed next to each physical plant before the smartphone is handed over to the user. The application then calculates which information should be displayed to the user based on the user's own position in the room, the distances to the plants, and the orientation of the device. In comparison to the LED prototype, no manual control of the user's position during the study is necessary. In the lower part of the display, a colored circle shows the overall status of the plants in the room.

\section{FIELD STUDY EXPLORING USER EXPERIENCE}

The overall goal of the (repeated measures) user study was to explore the user experience (UX) of the two systems, including whether the use of the systems has an effect on the wellbeing determinants autonomy, competence, and motivation.

\section{Participants and Apparatus}

We recruited 24 participants (12 females, 12 males) aged between 17 and $59(\mathrm{M}=28, \mathrm{SD}=11)$. All participants reported to have no color vision weakness and were familiar with the use of mobile devices. The participants rated their skills and interest in houseplant care with a 7-point Likert scale $(-3=$ beginner/unimportant, $3=$ professional/important) in average more than beginners $(M=-1,04, S D=1,68)$, but they indicated interest in plant care $(\mathrm{M}=0,38, \mathrm{SD}=1,58)$. The study was conducted in a living room to provide an environment where users normally interact with houseplants (see Figure 6). For each run, three plants were placed in the room at a distance of $2 m-3 m$ from each other. The watering can was placed on a table near the plants. The plants had different initial water levels (i.e., very low, low, sufficient) to show the user the different conditions that exist. For testing the AR system, an iPhone X was used to display the information in the AR condition.

\section{Questionnaires}

We chose the attrakDiff questionnaire to evaluate the different systems considering UX. The questionnaire measures pragmatic quality (PQ), hedonic quality (HQ) resulting from a combination of HQS and HQI, and overall attractiveness (ATT). HQS measures the perceived ability of a product to meet a person's desire for self-improvement, HQI measures the perceived ability of a product to communicate a valuable identity to others [18]. The attractiveDiff questionnaire is particularly suitable for our study as it focuses on hedonic qualities (with HQS, HQI, and ATT), which may play a greater role in ones own home than, for example, in a work environment. In addition, participants were asked to make a self-assessment of how the use of the respective system would affect the three positive computing factors motivation, competence, and autonomy on a 7-point Likert scale. Motivation and competence were chosen to explore whether users would be more motivated to take care of their plants with the systems and improve their skills. An effect on these two factors was measured with the questions "How do you think your motivation to water your plants would change with the use of each system" and "How do you think your own ability to give your plants the right amount of water would change with the use of each system" (strongly worsened, strongly improved). The question of autonomy was intended to check whether the system restricts users in their tasks. It was addressed with the question "Who has control of the watering process?" (the system, the user).

\section{Procedure}

We briefly described that the study aims to test two different systems, which help water houseplants. Before the participants tested the two systems one after another, they were asked to read a brief description of the system and of the task they would perform. The task was, with the support of the system, to water all three plants up to the required amount of water. After completing the task, they rated the attractiveness of the system using the attrakDiff questionnaire. This process was repeated for the second system. Afterwards, the participants answered questions about the positive computing factors. At the end, participants were asked which of the systems they preferred and had to give reasons for their preference. Each participant completed the study in about 30 minutes.

\section{Results}

First, the general trends in the "quantitative" data (collected with the questionnaires) are identified with the help of graphical representations, and then statistical analyses are performed to test for significance. Afterwards, we describe the results of the qualitative data analysis aiming to provide reasons for specific results and observations. 


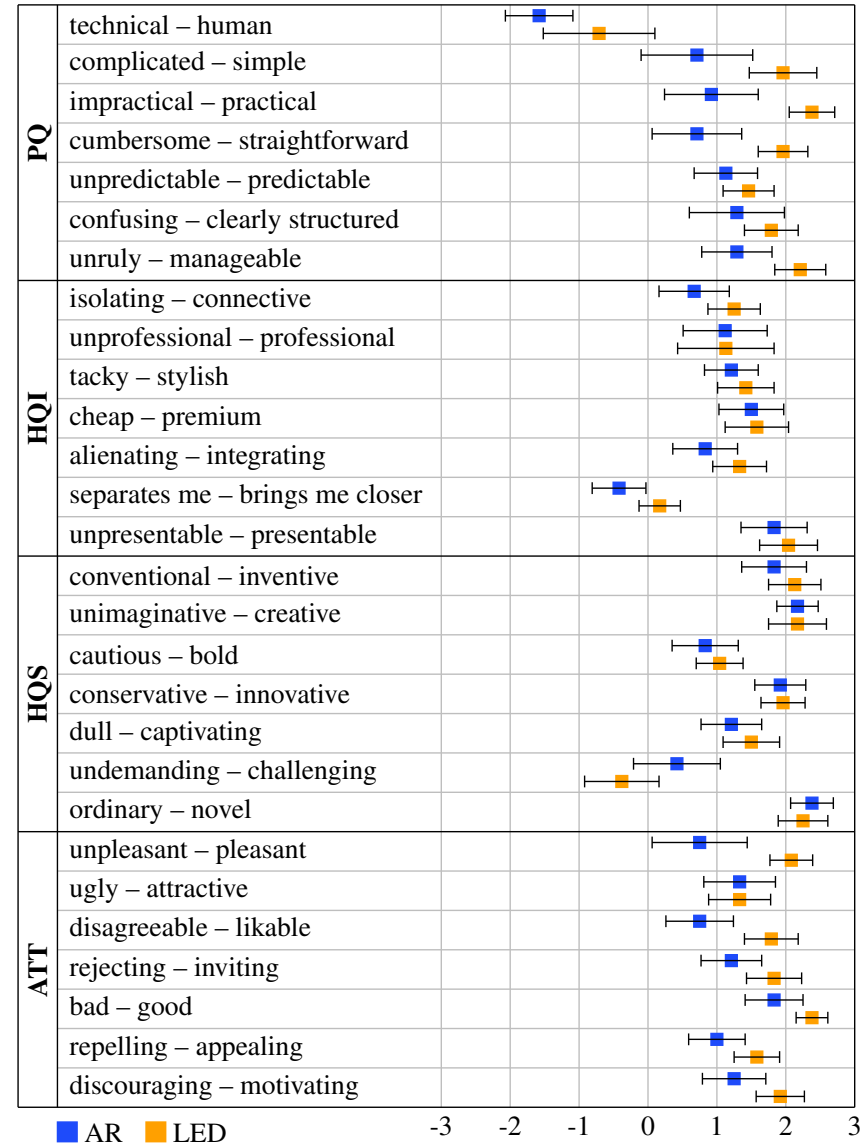

Figure 7. Overview of mean ratings for each item of the attrakDiff questionnaire. The items are sorted from left (negative) to right (positive). Error bars denote $95 \%$ confidence intervals.

\section{General Trends}

Figure 7 presents an overview of how each item of the attrakDiff was rated on average for both conditions. In most items, the LED system was rated better than the AR system. Especially with the items belonging to PQ, the LED system performs much better. The only exception where the AR system was rated clearly better is the item "undemandingchallenging", which is part of the HQS measurement. Figure 8 provides an overview of mean values for each of the independent variables measured by the attrakDiff questionnaire (i.e., PQ, HQI, HQS, ATT). As already indicated, the biggest differences can be seen in the PQ, where the LED prototype performs much better. It also has better results in HQI and ATT. Only in HQS both systems are rated almost equally. Figure 9 shows the mean values of the evaluation of the positive computing questions. Here, the trend continues that the LED system performs better.

\section{Statistical Analysis}

A paired-samples t-test was conducted to test the differences between the two systems (i.e., LED, AR) across the dependent variables measured by the attrakDiff questionnaire (i.e., PQ, HQI, HQS, ATT) and the three positive computing factors (i.e. motivation, competence, autonomy). The t-test showed a significant effect on the dependent variables mea-

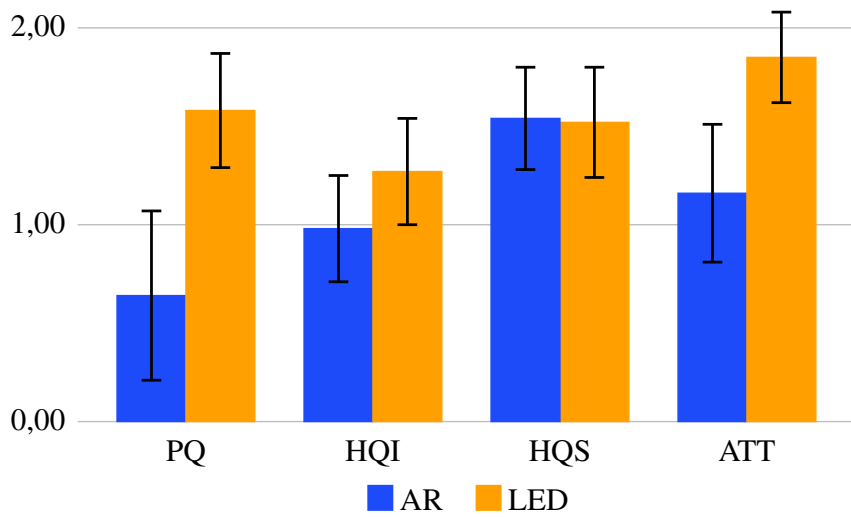

Figure 8. Overview of mean values for each condition and dependent variable measured by the attrakDiff questionnaire. Error bars denote $95 \%$ confidence intervals. Scale ranges from -3 to 3 .

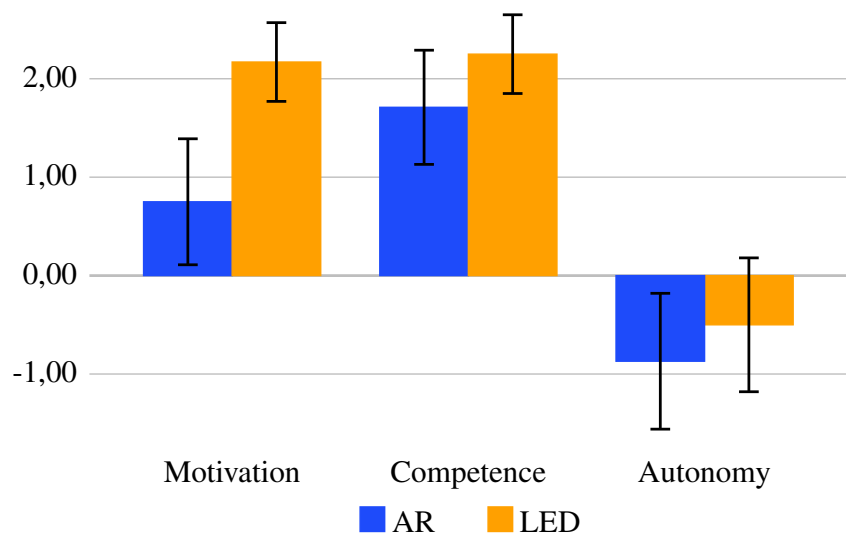

Figure 9. Overview of mean values for each of the three positive computing factors based on the participants' self-assessment. Error bars denote $95 \%$ confidence intervals. Scale ranges from -3 to 3 .

sured with the attrakDiff on PQ $\mathrm{t}(23)=4.711, \mathrm{p}<0.001$ and $\operatorname{ATT} t(23)=3.508, p=.002$. No significant effects were found on HQI $\mathrm{t}(23)=1.827, \mathrm{p}=.081$ and HQS $\mathrm{t}(23)=(-) 0.097$, $\mathrm{p}=0.924$. For the factors of positive computing, there was a significant effect on motivation $\mathrm{t}(23)=3.983, \mathrm{p}<0.001$. Competence $\mathrm{t}(23)=1.919, \mathrm{p}=0.067$ and autonomy $\mathrm{t}(23)=1.251$, $\mathrm{p}=0.224$ showed no significant effects.

\section{Analysis of qualitative data}

20 of the 24 participants (83\%) said they preferred the LED system. 17 of these 20 people perceived the AR system's smartphone as limiting and participants stated that "it is impractical to hold a watering can and a smartphone". Further reasons were that "looking through the screen [of the smartphone] is unreal" and that the LED system is "less complicated", "more intuitive" and "easier to use". Participants who preferred the AR system justified their selection by the fact that it is "possible to use any flower pots" and "the display is visible from all sides". It was also mentioned that you can decide for yourself when you want to see the display and thus have the possibility to improve your own abilities, for example, by using the display only for controlling and not during the complete watering process. 


\section{DISCUSSION}

In the beginning, we criticized a potential future with smarthomes in which many of todays routine tasks would be facilitated or even replaced by automation. We then motivated our research by pointing out that there are healthy routines in homes, such as watering houseplants, which should not be replaced carelessly through automation but interactions should be meaningfully transformed through appropriate design. Thus, our motivation was as suggested by Calvo and Peters [8] and other fellow researchers (e.g., [4, 26, 33]) being sensitive towards automation technology's positive and negative qualities, including undesired feelings of loss of control and smart things easily becoming obnoxious.

In order to design socio-spatially meaningful interactions, we applied role-play as a methodology and used the implicit interaction and proxemic interaction frameworks as analytical tools. These frameworks build on early work, such as Mark Weiser's ground braking work on "ubiquitous and calm computing" [46], which ultimately inspired embodied interaction design [12]. The implicit interaction framework helped to understand if, for example, plants should act proactive or reactive and the proxemic interaction framework helped to identify concrete measures, such as what distance and orientation to trigger actions. We found that iterating role-plays with participants and changing participants' interaction modalities, such as blindfolding participants or allowing participants only to communicate with a chat app useful. We believe to have provided the design process in detail and in a manner that it could be replicated by fellow researchers for other use cases considering interactions with non-human agents in smarthomes. While it was clear to us that embedded technology was needed to at least enable non-human agents to sense contextual information, to network, and communicate with other "smart" things, we were not sure about the output generated for users and which mode (and associated forms of interaction) would be best in terms of perceived attractiveness and potential impact on wellbeing determinants, such as motivation.

Considering the interaction modality or mode between an inhabitant and non-human agents in smarthomes, we were convinced that physical actuators (especially LEDs or LED based displays) and AR were todays ecologically valid alternative techniques and thus worth focusing our implementations and study on. Therefore, we implemented two systems considering these two alternative techniques and conducted a user study in the field comparing their benefits and limitations. We, ourselves were not convinced which alternative would be preferred for a houseplant watering scenario in smarthomes. However, it was clear to us that too many physical actuators, such as LEDs seemed unreasonable but not necessarily unmanageable by a rigorous design process. On the other side, we saw the potential material qualities of AR, such as its conditional existence, low maintenance, non-ephemeral (in a traditional sense) characteristic, which could benefit inhabitants and their wellbeing (if users had and felt control and competence to manage AR's conditional existence). Thus, the research question we aimed to address with the field study was which technique was preferred for watering houseplants in future smarthomes and why. Furthermore, we hoped to gain some insights on interaction modality preferences between non-human agents and inhabitants in smarthomes in general.

The results of the user study show that both prototypes were perceived as enrichment by potential users (see Figure 7), who saw a potential that the systems would increase their long-term motivation and may improve their own abilities without the users losing too much control (see Figure 9). Nevertheless, participants preferred the solution with embedded information directly in the flowerpot over the AR solution. The LED prototype was evaluated significantly better by participants considering perceived usability (PQ) and overall attractiveness (ATT). We found that participant disliked to have a smartphone in one hand and the watering can in the other and have to look through the smartphone display while interacting with plants. We deliberately chose a smartphone because users already have access to this type of augmented reality device. Headmounted displays, which might also be available to consumers in future, could increase the attractiveness and usefulness of AR solutions for such tasks, as hands would then be free.

The main limitations of our research are associated with possible long-term effects, which would be relevant for daily routines. The two positive computing factors motivation and competence may change in the long term. Without long-term user studies, it seems impossible to make concrete statements about how inhabitants' motivation and competence may change. Since we still wanted to know whether certain tendencies exist, we asked the participants for a self-assessment and an anticipation of potential futures. Furthermore, physical actuators such as LEDs may ware off, stop working, and may become less attractive over time in contrast to AR. It should also be noted that during the study we had to use the "Wizard-of-Oz" technique to simulate spatial interaction with the embedded system, because indoor localization is a difficult to implement task. However, AR is already space sensitive and it was easy to implement proximity sensitive functions. Therefore, we believe future designs might integrate both techniques.

\section{CONCLUSION}

We reported on a rigorous design process aiming to explore how IoT technology can support inhabitants in properly watering their plants in their homes without replacing the benefits inhabitants may have from routine interactions with their plants. We presented results from multiple role-plays, and a field study with concluding designs, comparing the user experience of two alternative techniques to implement (implicit and proxemic) interactions between inhabitants and their plants. We found, for example, that the user experience of IoT plants providing feedback through physical LEDs was significantly better than when plants used an AR solution with smartphones.

We hope that the detailed description of the design process we went through and the results we produced will inspire and guide fellow researchers in designing implicit interactions in future smarthomes and inform them of potential benefits and limitations of embedded physical actuators and AR to communicate (explicitly and implicitly) with inhabitants. 


\section{REFERENCES}

[1] Leonardo Angelini, Stefania Caparrotta, Omar Abou Khaled, and Elena Mugellini. 2016. EmotiPlant: Human-Plant Interaction for Older Adults. In Proceedings of the TEI'16: Tenth International Conference on Tangible, Embedded, and Embodied Interaction. ACM, 373-379.

[2] Constantinos Marios Angelopoulos, Sotiris Nikoletseas, and Georgios Constantinos Theofanopoulos. 2011. A Smart System for Garden Watering Using Wireless Sensor Networks. In Proceedings of the 9th ACM International Symposium on Mobility Management and Wireless Access (MobiWac '11). ACM, New York, NY, USA, 167-170. DOI :

http://dx.doi.org/10.1145/2069131.2069162

[3] Till Ballendat, Nicolai Marquardt, and Saul Greenberg. 2010. Proxemic interaction: designing for a proximity and orientation-aware environment. In $A C M$ International Conference on Interactive Tabletops and Surfaces. ACM, 121-130.

[4] Louise Barkhuus and Anind Dey. 2003. Is context-aware computing taking control away from the user? Three levels of interactivity examined. In International Conference on Ubiquitous Computing. Springer, 149-156.

[5] Virginia Braun and Victoria Clarke. 2006. Using thematic analysis in psychology. Qualitative research in psychology 3, 2 (2006), 77-101.

[6] A.J. Bernheim Brush, Bongshin Lee, Ratul Mahajan, Sharad Agarwal, Stefan Saroiu, and Colin Dixon. 2011. Home Automation in the Wild: Challenges and Opportunities. In Proceedings of the SIGCHI Conference on Human Factors in Computing Systems (CHI '11). ACM, New York, NY, USA, 2115-2124. DOI: http://dx.doi .org/10.1145/1978942.1979249

[7] Marion Buchenau and Jane Fulton Suri. 2000. Experience prototyping. In Proceedings of the $3 \mathrm{rd}$ conference on Designing interactive systems: processes, practices, methods, and techniques. ACM, 424-433.

[8] Rafael A Calvo and Dorian Peters. 2014. Positive computing: technology for wellbeing and human potential. MIT Press.

[9] S. Chang, S. Ham, and D. Suh. 2010. ROHINI: A robotic flower system for intuitive smart home interface. In ICCAS 2010. 1773-1776. DOI :

http://dx.doi.org/10.1109/ICCAS. 2010.5670207

[10] Jaewon Cho, Sanghoo Park, Been Jeon, Byung-Chull Bae, and Jun-Dong Cho. 2015. People's Emotional Responses to a Plant's Emotional Expression. In Proceedings of the Ninth International Conference on Tangible, Embedded, and Embodied Interaction. ACM, 545-550.

[11] Drashti Divani, Pallavi Patil, and Sunil K Punjabi. 2016. Automated plant Watering system. In Computation of Power, Energy Information and Commuincation (ICCPEIC), 2016 International Conference on. IEEE, 180-182.
[12] Paul Dourish. 2001. Where the action is. MIT press Cambridge.

[13] Berry Eggen, Gerard Hollemans, and Richard van de Sluis. 2003. Exploring and enhancing the home experience. Cognition, Technology \& Work 5, 1 (01 Apr 2003), 44-54. DOI :

http://dx.doi.org/10.1007/s10111-002-0114-7

[14] Berry Eggen, Elise van den Hoven, and Jacques Terken. 2017. Human-Centered Design and Smart Homes: How to Study and Design for the Home Experience? Springer International Publishing, Cham, 83-92. DOI :

http://dx.doi .org/10.1007/978-3-319-01583-5_6

[15] Marjolein Elings. 2006. People-plant interaction: the physiological, psychological and sociological effects of plants on people. In Farming for health. Springer, 43-55.

[16] Google. 2018. ARCore Overview. (2018). https://developers. google. com/ar/discover/.

[17] JC Hammond, PM Sharkey, and GT Foster. 1996. Integrating augmented reality with home systems. In Proc. 1 st Euro. Conf. Disability, Virtual Reality \& Assoc. Tech.

[18] Marc Hassenzahl, Michael Burmester, and Franz Koller. 2003. AttrakDiff: Ein Fragebogen zur Messung wahrgenommener hedonischer und pragmatischer Qualität. In Mensch \& Computer 2003. Springer, 187-196.

[19] Pam Heath and Nancy Bell. 2006. The Changing World of Home Technology: A Microsoft Case Study. Information Society 22, 4 (2006), 251 - 259.

[20] Steven Henderson and Steven Feiner. 2011. Exploring the benefits of augmented reality documentation for maintenance and repair. IEEE transactions on visualization and computer graphics 17, 10 (2011), 1355-1368.

[21] Steven J Henderson and Steven Feiner. 2009. Evaluating the benefits of augmented reality for task localization in maintenance of an armored personnel carrier turret. In Mixed and Augmented Reality, 2009. ISMAR 2009. 8th IEEE International Symposium on. IEEE, 135-144.

[22] Apple Inc. 2017. ARKit. (2017). https://developer.apple.com/arkit/.

[23] Lars-Erik Janlert and Erik Stolterman. 2017. Things that keep us busy: The elements of interaction.

[24] Wendy Ju. 2015. The design of implicit interactions. Synthesis Lectures on Human-Centered Informatics 8, 2 (2015), 1-93.

[25] Wendy Ju, Brian A Lee, and Scott R Klemmer. 2008. Range: exploring implicit interaction through electronic whiteboard design. In Proceedings of the 2008 ACM conference on Computer supported cooperative work. ACM, 17-26. 
[26] Wendy Ju and Larry Leifer. 2008. The design of implicit interactions: Making interactive systems less obnoxious. Design Issues 24, 3 (2008), 72-84.

[27] S. Koprda, Z. Balogh, D. Hrubý, and M. Turčám. 2015. Proposal of the irrigation system using low-cost Arduino system as part of a smart home. In 2015 IEEE 13th International Symposium on Intelligent Systems and Informatics (SISY). 229-233. DOI :

http://dx.doi.org/10.1109/SISY.2015.7325385

[28] Matthias Kranz, Paul Holleis, and Albrecht Schmidt. 2010. Embedded interaction: Interacting with the internet of things. IEEE internet computing 14, 2 (2010), 46-53.

[29] David Ledo, Saul Greenberg, Nicolai Marquardt, and Sebastian Boring. 2015. Proxemic-aware controls: Designing remote controls for ubiquitous computing ecologies. In Proceedings of the 17th International Conference on Human-Computer Interaction with Mobile Devices and Services. ACM, 187-198.

[30] Jangho Lee, Jee-In Kim, Jiyong Kim, and Ji-Young Kwak. 2007. A unified remote console based on augmented reality in a home network environment. In Consumer Electronics, 2007. ICCE 2007. Digest of Technical Papers. International Conference on. IEEE, $1-2$.

[31] Rung-Huei Liang. 2012. Designing for unexpected encounters with digital products: Case studies of serendipity as felt experience. International Journal of Design 6, 1 (2012).

[32] Nicolai Marquardt and Saul Greenberg. 2015. Proxemic interactions: From theory to practice. Synthesis Lectures on Human-Centered Informatics 8, 1 (2015), 1-199.

[33] Sarah Mennicken, Jo Vermeulen, and Elaine M Huang. 2014. From today's augmented houses to tomorrow's smart homes: new directions for home automation research. In Proceedings of the 2014 ACM International Joint Conference on Pervasive and Ubiquitous Computing. ACM, 105-115.

[34] Microsoft. 2017. HoloLens. (2017). https://www.microsoft.com/en-us/hololens.

[35] Paul Milgram, Haruo Takemura, Akira Utsumi, and Fumio Kishino. 1995. Augmented reality: A class of displays on the reality-virtuality continuum. In Telemanipulator and telepresence technologies, Vol. 2351. International Society for Optics and Photonics, 282-293.

[36] Lars Müller, Ilhan Aslan, and Lucas Krüßen. 2013. GuideMe: A Mobile Augmented Reality System to Display User Manuals for Home Appliances. In Advances in Computer Entertainment, Dennis Reidsma, Haruhiro Katayose, and Anton Nijholt (Eds.). Springer International Publishing, Cham, 152-167.
[37] Dan O'Sullivan and Tom Igoe. 2004. Physical computing: sensing and controlling the physical world with computers. Thomson Boston, MA, USA.

[38] Parrot Drones SAS. 2015. Parrot Smart Pot. (2015). https:

//www.parrot.com/uk/connected-garden/parrot-pot.

[39] Andreas Seiderer, Chi Tai Dang, and Elisabeth André. 2017. Exploring Opportunistic Ambient Notifications in the Smart Home to Enhance Quality of Live. In Enhanced Quality of Life and Smart Living. Springer International Publishing, Cham, 151-160.

[40] Dipak Surie, Berker Baydan, and Helena Lindgren. 2013. proxemics awareness in kitchen As-A-Pal: tracking objects and human in perspective. In Intelligent Environments (IE), 2013 9th International Conference on. IEEE, 157-164.

[41] Arthur Tang, Charles Owen, Frank Biocca, and Weimin Mou. 2003. Comparative effectiveness of augmented reality in object assembly. In Proceedings of the SIGCHI conference on Human factors in computing systems. ACM, 73-80.

[42] Brent Tisserat and Carl E Vandercook. 1985. Development of an automated plant culture system. Plant cell, tissue and organ culture 5, 2 (1985), 107-117.

[43] Ahmed Mohmmad Ullah, Md Rashedul Islam, Sayeda Farzana Aktar, and SK Alamgir Hossain. 2012. Remote-touch: Augmented reality based marker tracking for smart home control. In Computer and Information Technology (ICCIT), 2012 15th International Conference on. IEEE, 473-477.

[44] Alladi Venkatesh. 1996. Computers and Other Interactive Technologies for the Home. Commun. ACM 39, 12 (Dec. 1996), 47-54. DOI : http://dx.doi.org/10.1145/240483.240491

[45] Alexandra Voit, Tonja Machulla, Dominik Weber, Valentin Schwind, Stefan Schneegass, and Niels Henze. 2016. Exploring Notifications in Smart Home Environments. In Proceedings of the 18th International Conference on Human-Computer Interaction with Mobile Devices and Services Adjunct (MobileHCI '16). ACM, New York, NY, USA, 942-947. DOI : http://dx.doi .org/10.1145/2957265.2962661

[46] Mark Weiser. 1991. The Computer for the 21 st Century. Scientific american 265, 3 (1991), 94-105.

[47] Charlie Wilson, Tom Hargreaves, and Richard Hauxwell-Baldwin. 2015. Smart homes and their users: a systematic analysis and key challenges. Personal and Ubiquitous Computing 19, 2 (01 Feb 2015), 463-476. DOI: http://dx.doi.org/10.1007/s00779-014-0813-0 